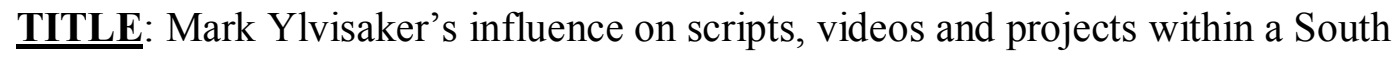
African context

Author: Glenn Goldblum, D.Phil

Department of Communication Pathology

University of Pretoria

Pretoria

South Africa

E-mail: glenngol@netactive.co.za

\title{
Mailing address:
}

Department of Communication Pathology

University of Pretoria

Pretoria

South Africa

0002

Phone: +South African Code: 114400087

Mobile: +South African Code 824120136

Fax: +South African Code: 118879081 


\begin{abstract}
The current article overviews a range of collaborative group projects undertaken by members of the Conversation Groups for individuals living with aphasia and cognitive-communication disorders in the Department of Communication Pathology, University of Pretoria, South Africa. Projects work towards creating networks of support, and lessening barriers for the individual living with aphasia and cognitivecommunication disorders. The article highlights the pervasive and far-reaching influence across continents of Professor Mark Ylvisaker's philosophy, writings and personal teachings on both Conversation Group projects, as well as student clinician training practice in the University of Pretoria context. In addition, Ylvisaker's influence is described on the development of a collaborative communication partner training program within a South African retail supermarket environment, highlighting the effort to remove barriers between employees and customers with a cognitivecommunication disability specifically.
\end{abstract}

KEYWORDS: Mark Ylvisaker; conversation group; aphasia; cognitivecommunication disorders; barriers 
Conversation Group (CG) projects for individuals living with aphasia and cognitivecommunication disorders in the Department of Communication Pathology, University of Pretoria (UP), South Africa have been philosophically inspired by the teachings and input of Professor Mark Ylvisaker since their inception in 1995. I started corresponding with him personally in 1996, and had the privilege of meeting him for the first time in Schenectady, NY in 2002. Until 2009, in addition to his publications, I was regularly guided and mentored by him with his personal philosophy, observations and teachings - some of which have been included below. Living on another continent, the time and effort taken by Professor Ylvisaker for personal input and guidance has always been deeply appreciated. It is a great honor and privilege to be invited to make a contribution to this special tribute issue.

"Rehabilitation....is more than the mechanical application of technical procedures. In our judgment, it involves a commitment to enter the lives of the people with disability, to create collaborative partnerships with them and the everyday people in their lives, and to support them in part by serving as an ongoing source of optimism, creativity, flexibility, and enthusiasm in the face of the obstacles that often seem overwhelming"(p.xi). ${ }^{1}$

These words cohesively reflect Mark Ylvisaker's philosophical and humanitarian legacy - a legacy that has profoundly impacted brain injury rehabilitation practice globally, and my personal rehabilitation priorities specifically when working with individuals with brain injury in South Africa. The following article attempts to highlight this impact. 
Paradigm shifts within the rehabilitation context, specifically in relation to individuals with neurogenic-based communication disorders have been advocated by the participation-based International Classification of Functioning, Disability and Health framework (ICF) ${ }^{2}$ combined with consumer-driven intervention models including the social model of disability; ${ }^{3-5}$ the Life Participation Approach to Aphasia Project Group (LPAA) ${ }^{6}$ and the supported participation model. ${ }^{7}$ These models propose moving beyond creating functional and relevant outcomes for the individual with communication disorders, to creating environmentally-and-communication-friendly places, with fewer barriers and more facilitators.

With regard to the rehabilitation of individuals with chronic cognitive behavioral and communication impairments after brain injury, numerous authors ${ }^{7-16}$ have advocated a collaborative brain injury intervention approach using an apprenticeship or 'supported participation' model'(p.9). ${ }^{7}$ In this functional and richly contextualized approach, everyday people collaborate with, and provide ongoing supports for the individual with a traumatic brain injury (TBI) to participate within the context of their everyday routines (including the home, work, school environment and service encounters). Collaboration ranges from communication partner training programs, to situational coaching of both the individual with a TBI and the relevant person in a particular context (e.g. the teacher, parent, therapist, aide, employer and service provider) about ways to use positive behaviour supports and become "facilitative conversationalists"(p.787). ${ }^{16}$ Through this, endeavoring to elicit positive, respectful communicative interaction from, and enhanced participation for, the individual with a $\mathrm{TBI}^{17,18}$ thereby empower both individuals with a communication disorder and their communication partners. 
Specifically, Ylvisaker \& Feeney ${ }^{19-21}$ and Ylvisaker et al. ${ }^{22}$ advocate the use of projects taking the form of, for example, collaboratively-produced self-advocacy videos that ideally create an expert role for the person with a disability. This is empowering both for that individual, as well as for their audiences, who in turn gain more competence in interacting with person with the disability. In addition, through working repeatedly on goal setting, planning and monitoring with support by others in everyday contexts, practice becomes increasingly automatic and strategies become increasingly internalized. ${ }^{7,23}$ This helps individuals to succeed at levels "beyond those predicted by their degree of neurologic impairment"(p.223). ${ }^{13}$ Not only has this collaborative/supported participation approach within the individual's own culture and context been viewed by Ylvisaker and Feeney ${ }^{20}$ and Ylvisaker et al. ${ }^{7}$ as working towards the goal of a more meaningful and ultimately satisfying life for the individual, but with reference to the $\mathrm{ICF}^{2}$ this approach can be conceptualized as removing social barriers and improving their ability to participate more deeply and in a more sustainable way in their everyday communities. ${ }^{24-26}$

Ylvisaker's thinking and philosophy have been woven through the range of projects undertaken by group members of the two Conversation Groups (CG) for persons living with aphasia (PwA) and persons with cognitive communication disorders (PwCCD) respectively at the UP since the groups' inception in 1995, and has also had an impact on student clinician training. The current article provides an overview of these projects. It also describes the impact of Ylvisaker's influence on the development of a collaborative communication partner training program within a South African retail supermarket environment. ${ }^{8-10}$ 


\section{Conversation Groups for Individuals living with aphasia and cognitive-}

communication Disorders, University of Pretoria: An overview of practical issues The CG for individuals living with chronic aphasia and cognitive-communication disorders (comprising a group for PwA, and a group for PwCCD) were established by the author in 1995 in the Speech and Hearing Clinic of the Department of Communication Pathology, UP. They continue to meet separately or in combination, depending on the projects undertaken at the time. Each CG is facilitated by three final year Communication Pathology student clinicians respectively under my supervision. CG comprises group members who are English, Afrikaans and Black first-languagespeakers. CG numbers have varied over the years with an average of 8 members in the group for PwA; and 10 members in the group for PwCCD. The CG meet once weekly for $1 \frac{1}{2}$ hours during the University term.

\section{CG Assessment protocols: 1995 - 2009}

Since 1995, various assessment protocols have been used, focusing in particular on the communication interaction and participation of the group members within the group over time, with an emphasis on qualitative and subjective ratings by $\mathrm{CG}$ members of various projects undertaken. These measures have included the Pragmatic Protocol, ${ }^{27}$ and in 1999, a pilot Quality of Life (QOL) Scale, which was developed by Goldblum, Mulder and Von Gruenewaldt ${ }^{28}$ to determine the perceived effect of group therapy on the QOL and social communication competence of each group member. This QOL Scale was replaced in 2007 by the Communication Interaction Rating Scale for Aphasia Group (CIRSAG), ${ }^{29}$ an informal rating scale designed to assess an individual's interaction skills in an aphasia group. 


\section{CG: An overview of philosophy and goals}

CG goals reflect the above-described paradigm shifts. ${ }^{2-7}$ Goals are formulated under the umbrella of advocacy, empowerment and real engagement, ${ }^{30,31}$ aiming to dismantle and lessen barriers both personally for the individual, as well as on a broader societal level, working towards real engagement in life activities in spite of residual communication difficulties.

CG goals focus on getting the message across with the necessary support, rather than on communication perfection and linguistic accuracy. Empowerment principles guide these efforts where CG members, within their capabilities, collaborate with their student clinicians, in brainstorming and setting goals for projects; implementing the projects and where appropriate, evaluating their outcomes. ${ }^{32}$ Here, the PwA and PwCCD work towards collaborating with everyday people in their lives who will then hopefully provide them with ongoing supports, facilitating their fuller participation in their daily lives. ${ }^{7,13-15,20}$ These projects also provide the CG members the opportunity to attempt to lessen or even dismantle barriers for PwA and PwCCD, endeavoring to elicit positive and respectful communicative interaction for them. ${ }^{17,33}$

\section{Projects undertaken by the Conversation Groups of the University of Pretoria,}

\section{Department of Communication Pathology}

"Projects ideally create an expert role for the person with a disability, thereby helping the person transform a self-concept dominated by a sense of incompetence" (p. 2$).^{34}$

Since 1995, CG members have actively participated in the formulation and implementation of a range of self-advocacy projects that have either been individually 
identified and worked on by the individual CG members, or worked on as a group project by the PwA and PwCCD either in their separate groups, or in combination. Ylvisaker in a personal communication, dated October 14, 2001 noted: "I am still enthusiastic about ensuring meaningful themes in ones work with people.... especially disorganized people who may not easily see the point of therapy activities. But increasingly I am attracted to the idea of engaging folks in projects that have an actual product - especially a project/ in which the individual can be considered an expert contributor and a product that will benefit somebody."

CG projects have evolved over time to reflect the changing interests, needs and priorities of the CG members. In most of the following projects, either individual CG members, or the whole group are encouraged to actively participate, thereby clarifying their thinking and often writing their own script in relation to the topic under discussion. The benefit of this has been highlighted by Jason who stated that "The concept of developing scripts for tough situations work. This is the one way for people with brain injury to take control of their lives" (p. 265). ${ }^{35}$

Individually-focused projects have included among others, writing motivational letters to the press; writing talks for school children and rehabilitation groups; information-sharing with employers and colleagues; developing personal web pages and business plans. One CG member worked within the group context on his dream of developing the first brain injury support group in South Africa, which in 2000 became a reality, called the Brain Injury Group (BIG). In 2002, he led BIG supporters on a march in Pretoria to the National Lottery Board asking them to fund his project! Group-constructed projects began in 1997 when the group members collaboratively began to create their ground rules or constitution guiding group members and clinicians about appropriate and inappropriate group behaviour. These are revised 
annually and are helpful to student clinicians in particular. In 1997, through the request of various CG members, the first Open Evening was established - a community-education project that has become the annual highlight of the year for the CG members, where family members, friends, interested members of the public and students attend, and the projects worked on through the year are showcased. From a small beginning in a lecture room in the UP Speech and Hearing Clinic, this has now evolved into a large event, where a conference room is hired on the UP campus to accommodate the 120 or more people attending. Over the years the Open Evening has become a large collaborative project where student clinicians and CG members work diligently on presenting their projects in a cohesive manner.

In the ongoing effort to dismantle barriers, CG members (supported by their clinicians) have formulated public awareness-raising brochures in English and Afrikaans. These brochures provide information and suggestions for families and employers on how to communicate with PwA and PwCCD. In 2003 and 2004,,$^{36,37}$ CG members made videos of their fellow group members presenting the various CG projects, and then showed them at our annual Open Evening. Among these, CG members made self advocacy videos reflecting on issues around their being entrenched as a result of having aphasia, where they formulated their own feedback to their employers under these very difficult circumstances. Scripts were collaboratively written with the clinicians.

In $2004^{37} \mathrm{CG}$ members listened to a reading of a diversity awareness fable, The Giraffe and the Elephant, ${ }^{38}$ and analyzed and re-wrote the fable in their own words. CG members spent time interpreting the issues raised in the fable in relation to the 
obstacles they encountered living with aphasia and cognitive-communication disorders. Armed with their video cameras, student clinicians accompanied CG members into their homes, as well as onto the UP campus, identifying physical, communication and attitudinal obstacles in these contexts. Suggestions were then made as to how to overcome these barriers. The process and outcome of this project was presented at the $2^{\text {nd }}$ Southern African Neurological Rehabilitation Conference (SANRA), Johannesburg, South Africa, February, 2005 where Professor Ylvisaker was the Invited International Keynote speaker.

CG members have prepared and presented talks to participate in Toastmaster groups (Kagan: Toastmasters International Aphasia Gavel Club, Aphasia Institute, Toronto); and have also actively participated in a book club group, ${ }^{39}$ reading the book Tuesdays with Morrie $^{40}$ where some CG members prepared additional comments and materials to share with the groups weekly. Over the years, as advocated by Ross, Winslow and Marchant, ${ }^{41}$ experts have also been invited as resources to visit the $\mathrm{CG}$, sharing their expertise on a range of topics, including employment issues and challenges for individuals with disability in the workplace; professional story tellers, and a professional dance choreographer encouraging the group members to sing, dance and to re-connect with life.

In keeping with the technological advances, and the contemporary move towards digital social networking (DSN), another collaborative project is the CG's participation in an international digital networking project facilitating participation and learning by PwA and PwCCD as well as clinicians. In 2008, CG members from the UP collaborated in a project with PwA from the Aphasia Treatment Program at 
California State University, East Bay (CSUEB) using e-mail communication between these groups, exchanging photographs and information by both independent e-mailing group members, as well as those supported by their clinicians. ${ }^{42}$ In 2009 , this project evolved into a pilot 6 week Facebook $^{\text {TM }}$ Project between the 2 participating University group programs using Facebook ${ }^{\mathrm{TM}}$ as a mainstream social network

interface. ${ }^{43}$ Student clinicians facilitated creation of Facebook ${ }^{\mathrm{TM}}$ postings by PwA and PwCCD on 6 weekly discussion topics created by the project directors. Pre-postproject surveys were formulated and administered to the clinicians as well as the group participants about internet and social networking familiarity and use. Many anecdotal lessons were learned from this project including the necessity for support to be given to both clinicians as well as group participants. Facebook ${ }^{\mathrm{TM}}$ postings for PwA and PwCCD require different skills than private e-mail postings. Comments from group participants reflected their positive as well as negative perceptions about the project: "It's part of the way the world's going and we need to understand it" "I see Facebook ${ }^{\mathrm{TM}}$ as a public diary. What's the point?"

In view of the mostly positive anecdotal evidence noted by the participants - including reported increase in e-mailing by group participants, enjoyment of cross-cultural learning and friendships formed with other PwA and PwCCD across continents, expansions and refinements to this project have been proposed. ${ }^{43}$

\section{Observations and outcomes}

While many CG projects have been completed, many have not, and are either abandoned, or re-worked on at a later stage. Personal communication with Mark Ylvisaker (July 24, 2001) was reassuring in this regard: "The nice thing about 
projects is that the process is typically more important than the product-so if they never get entirely done, that's not necessarily a tragedy." The process of working on these projects was found to support the aims highlighted by Sohlberg ${ }^{44}$ and Ylvisaker $^{34}$ - namely to be empowering, providing the CG members the opportunity to thoroughly work on their executive skill functioning through problem analysis, the use of considerable planning and organizational skills, combined with a meaningful context for using their language and communication skills. Furthermore, CG members have varied in their ability to work independently on projects, and in the level of support needed for various projects. ${ }^{36}$ Ylvisaker (personal communication October 25, 2002) likewise noted that "differing levels of support are needed in developing projects for individuals functioning at different levels". In 2001, Goldblum et al. ${ }^{28}$ examined the impact of participation in a CG for PwCCD in the Department of Communication Pathology, UP over the time period 1995- 2000. Information was obtained through clinical observation over this six year period, combined with data gathered from the Pragmatic Protocol, ${ }^{27}$ and a pilot Quality of Life Scale compiled by the investigators, and administered to both the CG members as well as their significant others. Findings showed that in spite of the plateauing of pragmatic competence over time, the impact of CG therapy appeared to be reflected in perceived improvements in social-communicative competence and QOL. Significant others' perceptions of their family members' social-communicative competence and QOL were also more optimistic. The CG was perceived as therapeutic and supportive, resulting in improved insight and self confidence in the CG member. These findings were considered to lend support to Holland ${ }^{45}$ and Holland and Ross's ${ }^{46}$ reference to the inherent power of groups. Furthermore, Goldblum et al. ${ }^{28}$ concluded that in spite of the challenges of providing group therapy, with the implicit commitment of the 
group members to participating and sharing, "the resulting benefits will include, among others a sense of empowerment, a sense of greater control over their own lives with a concomitant increase in personal self-esteem" (p. 14). Goldblum et al. ${ }^{28}$ also concurred with others including Brown, Gordon and Haddad, ${ }^{48}$ and Whiteneck ${ }^{49}$ about the importance of using subjective perceptions in "filling the gaps by traditional measures" (p. 14), ${ }^{28}$ advocating the need to develop, refine and use subjective and QOL scales for our clinical interventions.

Over the years CG members have rated various projects using scales of 0-5 determining their enjoyment of, and perceived benefit from participating in identified projects. It has become increasingly clear that not everyone enjoys the same projects and this provides the clinicians with further insight and challenges when considering current and future projects and goals. While, for example Toastmaster Group (Kagan: Toastmasters International Aphasia Gavel Club, Aphasia Institute, Toronto) and Book $\mathrm{Club}^{39}$ group participation obtained an overall high rating of enjoyment $(85 \%)$ by the CG members respectively, some indicated their reluctance to participate in these activities in future.

In addition to using rating scales where appropriate, CG participants are always asked for their subjective comments prior to, and after completion of various projects. These comments often provide the most powerful anecdotal evidence supporting the worthiness of the projects undertaken, and the clear sense of community and friendship provided by CG participation - the inherent magic and power of groups referred to by Holland and Ross. ${ }^{46}$ Comments from members include: "Informing and educating the public in South Africa is important and also very exciting" 
"School kids seem to sit so quietly and you can hear a pin drop when they listen to my talk. It is amazing!"

"One of the most important things about the group is friends. They laugh with you, they comfort you, and most importantly, they tell you when it's not good enough." "A home away from home" (This feedback was written on Lightwriter ${ }^{\circledR}{ }^{47}$ by one of the CG participants using augmentative and alternative communication as his means of communication.)

Another outcome of CG projects has been the production of a pilot Shop Assist Card developed by CG members in collaboration with a large national South African retail supermarket store. The project involved CG members writing scripts in collaboration with their clinicians and then making videos to invite representatives of the company to meet with them. On meeting, they brainstormed ways to assist customers with disabilities in making shopping a less barrier-filled experience. These suggestions were then refined into a Shop Assist Card, which was eventually piloted by the supermarket in 2001. This CG project laid the foundation for access to later implementing a communication partner training program by Goldblum, ${ }^{8}$ and Goldblum \& Alant ${ }^{9,10}$ in the same supermarket chain as described below.

\section{Communication partner training program for sales assistants serving customers} with a TBI

The rationale for this communication partner training program developed out of the pervasive attitudinal and environmental barriers that exist regarding neurogenic communication disorders generally, and cognitive-communication disorders following a TBI specifically. ${ }^{8-10}$ Few published communication partner training programs are evident for TBI, in spite of collaborative communication partner training 
programs being advocated with uninformed sectors of society by Togher et al. ${ }^{12}$ to facilitate participation for individuals with TBI across a range of service encounters. Goldblum, ${ }^{8}$ and Goldblum and Alant ${ }^{9,10}$ developed and refined a four hour communication partner training session based on contemporary models of adult learning principles. The training was aimed at sales assistants in the large national South African supermarket chain mentioned above, in an effort to promote participation of people with TBI in the social world by reducing barriers through training. The ability of sales assistants to identify barriers to, and facilitators of, interactions involving customers with cognitive-communication disorders was investigated. In line with Ylvisaker's thinking ${ }^{7,16,17,33}$ the development of training and evaluation materials for this project included widespread consultation and collaboration with individuals with a TBI. The research assistant was an individual with a TBI, and individuals with a TBI assisted the researcher in all stages of the study - including initial focus groups, as well as helping to develop a set of realistic video scenarios for use in the main study. The study comprised an innovative, preliminary effort to provide experimental data for a participation-based, communication partner training program for the brain injured population, with positive outcomes even after a once-off training session. ${ }^{9,10}$ Furthermore, Goldblum, ${ }^{8}$ and Goldblum and Alant ${ }^{9,10}$ conclude that these results support thinking advocated by Ylvisaker, ${ }^{17,33}$ and Ylvisaker et al. ${ }^{7,16}$ among others, that "rehabilitation professionals should develop collaborative networks of support across social contexts." Such communication partner training programs will create "more facilitative and less barrier-filled communication opportunities for such individuals" ( p.104). ${ }^{10}$ 


\section{Conclusion}

Ripples of Professor Mark Yvlisaker's thinking, writings and teachings have extended from the College of Saint Rose in Albany, NY, USA to the University of Pretoria, South Africa. The far-reaching impact of his writings and personal input have been highlighted in describing the range of collaborative projects undertaken by CG members from the inception of the these groups at Pretoria University in 1995 until the present; as well as on student clinician training practice within this setting. In addition, Ylvisaker's influence on the development of a communication partner training project ${ }^{8-10}$ within a South African retail supermarket environment is described. All these projects are essentially collaborative in nature, ultimately aiming to create more environmentally-and-communicatively-friendly places with fewer barriers, more facilitators and networks of support in place for the PwA and the PwCCD. ${ }^{7}$

\section{Personal Postscript:}

On the $5^{\text {th }}$ May, 2009, less than 3 weeks before Mark Ylvisaker passed away, I emailed him, enquiring about his health. He e-mailed an immediate reply saying how ill he was, and with a few personal farewell lines. The only way I knew to say goodbye was to send him a photo of a bucket filled with my garden roses, inviting him to "smell the roses." His reply was almost immediate: "Beautiful roses - nice work."

This article is dedicated to the memory of my video-carrying friend, Mark Ylvisaker, whose philosophical teachings, generous mentorship and above all, special friendship, helped over many years to focus my rehabilitation priorities in South Africa, while collaborating with individuals with neurogenic-based communication disorders. 
He always had time to smell the roses.

\section{Acknowledgements}

The CG members for their courage, resilience and many life lessons they've been teaching us weekly since 1995

The student clinicians of the CG for individuals with aphasia and cognitivecommunication disorders, Department of Communication Pathology, University of Pretoria from 1995 to the present for their dedication, commitment and willingness to learn

Professor Erna Alant, Professor, Otting Endowed chair in Special Education University of Bloomington, Indiana for her guidance and support in the research examining sales assistants serving customers with traumatic brain injury that served as the author's D.Phil dissertation study.

Professor Janet Patterson, Department of Communicative Sciences and Disorders

California State University, East Bay; and VA Northern California Health Care System for her collaboration on the Facebook Project. 


\section{REFERENCES}

1. Ylvisaker M, Feeney TJ. Preface. In: Ylvisaker M, Feeney TJ. Collaborative brain injury intervention: Positive everyday routines. San Diego, CA: Singular; 1998: ix-xi

2. World Health Organization. ICF: International classification of functioning disability and health. Geneva, Switzerland: World Health Organization; 2001

3. French S. What is disability? In: French S, ed. On equal terms: Working with disabled people. London: Butterworth-Heinemann; 1994:3-16

4. Jordan L, Kaiser W. Aphasia: A social approach. London: Chapman \& Hall; 1996

5. Oliver M. Understanding disability: From theory to practice. London: Macmillan; 1996

6. LPAA Project Group: Chapey R, Duchan J, Elman R, Garcia L, Kagan A, Lyon J, Simmons-Mackie N. Life participation approach to aphasia: A statement of values for the future. ASHA Leader 2000; 4-6

7. Ylvisaker M, Jacobs H, Feeney TJ. Positive supports for people who experience disability following brain injury: A review. Journal of Head Trauma Rehabilitation 2003; 18(1): 7-32

8. Goldblum G. Sales assistants dealing with customers with Traumatic Brain Injury. Unpublished doctoral dissertation, University of Pretoria, South Africa 2005

9. Goldblum G, Alant E. Sales assistants serving customers with a traumatic brain injury. Poster presentation at the $37^{\text {th }}$ Annual Clinical Aphasiology Conference Scottsdale Arizona; May 2007

10. Goldblum G, Alant E. Sales assistants serving customers with a traumatic brain injury. Aphasiology 2008; 23(1): 87-109

11. Sohlberg MM, Glang A, Todis B. Improvement during baseline: three case studies encouraging collaborative research when evaluating caregiver training. Brain 
Injury 1998; 12(4): 333-346

12. Togher L, McDonald S, Code C, Grant S. Training communication partners of people with traumatic brain injury: A randomized controlled trial. Aphasiology 2004; 18(4): 313-335

13. Ylvisaker M, Feeney TJ. Executive functions after traumatic brain injury: Supported cognition and self-advocacy. Seminars in Speech and Language $1996 ; 17(3): 217-232$

14. Ylvisaker M, Feeney TJ. Supported behaviour and supported cognition: An integrated positive approach to serving students with disabilities. Educational Psychology in Scotland 2001; 6: 17-30

15. Ylvisaker M, Holland AL. Coaching self coaching and rehabilitation of head injury. In: Johns DF, ed. Clinical management of neurogenic communication disorders. Boston: Little Brown \& Co; 1985: 243-257

16. Ylvisaker M, Szekeres S, Feeney TJ. Communication Disorders associated with traumatic brain injury. In: Chapey R, ed. Language intervention strategies in aphasia and related neurogenic communication disorders. 4th ed. Baltimore MD: Lippincott Williams and Wilkins; 2001:745-808

17. Ylvisaker M. Intervention for individuals with challenging behaviour after TBI. Perspectives on Neurophysiology and Neurogenic Speech and Language Disorders: ASHA Division 2: 2002; 12(3):19-24

18. Ylvisaker M. Context-sensitive cognitive rehabilitation after brain injury: Theory and practice. Brain Impairment 2003; 4(1):1-16 19. Ylvisaker M, Feeney TJ. Collaboration and apprenticeship: Creating a network of competence, confidence and support. In: Ylvisaker M, Feeney TJ. 
Collaborative brain injury intervention: Positive everyday routines. San Diego: Singular; 1998:235-256

20. Ylvisaker M, Feeney TJ. Introduction to functional, everyday intervention:

Theory, research, and practice. In: Ylvisaker M, Feeney TJ. Collaborative brain injury intervention: Positive everyday routines. San Diego: Singular; 1998: 1-39

21. Ylvisaker M, Feeney TJ. The project approach in brain injury rehabilitation:

Theory and illustrations. Mini-seminar presented at the American Speech-LanguageHearing Association Annual Convention Washington DC; November 2000

22. Ylvisaker M, Todis B, Glang A, Urbanczyk B, Franklin C, DePompei

R, Feeney T, Maxwell NM, Pearson S, Tyler JS. Educating students with TBI:

Themes and recommendations. Journal of Head Trauma Rehabilitation 2001; $16(1): 76-93$

23. Ylvisaker M, DeBonis D. Executive function impairment in adolescence: TBI and ADHD. Top Lang Disord 2000; 20(2): 29-57

24. Alant E. Intervention and sustainability: Some issues. Paper presented at the $13^{\text {th }}$ Multi-professional AAC Research Indaba University of Pretoria South Africa; March 2005

25. Alant E. Support-based AAC intervention. In: Alant E, Lloyd L, eds. Augmentative and alternative communication and severe disabilities: Beyond poverty. London: Whurr; 2005:155-191

26. Alant E, Lloyd L. Epilogue. In: Alant E, Lloyd L, eds. Augmentative and alternative communication and severe disabilities: Beyond poverty. London: Whurr; 2005: 344-349 
27. Pruttting C, Kirchner D. Pragmatic aspects of language. JSHD 1987; 52: 105-119

28. Goldblum G, Mulder M, Von Gruenewaldt A. The process and power of group therapy for individuals with a closed head injury: An examination of the impact of participation in a conversation group for individuals with a closed head injury. The South African Journal of Communication Disorders 2001; 48: 3- 20 29. Garrett K, Staltari C, Moir L, Sitter, M. Communication Interaction Rating Scale for Aphasia Group (CIRSAG). In: Elman RJ, ed. Group treatment of neurogenic communication disorders: The expert clinician's approach. $2^{\text {nd }}$ ed.UK: Plural; 2007: $40-42$

30. Parr S, Duchan J, Pound C. Setting the scene. In: Parr S, Duchan J, Pound C. eds. Aphasia Inside Out. UK: McGraw Hill; 2003:1-9

31. Penman T, De Mare T. Changing places: reflections of therapists and group members on the power and potential of groups. In: Parr S, Duchan J, Pound C. eds. Aphasia Inside Out. UK: McGraw Hill; 2003:91-102

32. Sohlberg MM, Mateer CA. Introduction to cognitive rehabilitation. In: Sohlberg MM, Mateer CA. Cognitive rehabilitation: An integrative neuropsychological approach. New York: The Guilford Press; 2001: 3-24

33. Ylvisaker, M. Context-sensitive cognitive rehabilitation after brain injury: Theory and practice. Brain Impairment 2003;4(1): 1-16

34. Ylvisaker M. Ongoing project oriented intervention for children and adults with disability: collaboration and apprenticeship. Mini-seminar presented at the American Speech-Language-Hearing Association Annual Convention Washington DC; November 2000

35. Ylvisaker M, Feeney TJ. Epilogue. In: Ylvisaker M, Feeney TJ. Collaborative brain injury intervention: Positive everyday routines. San Diego: Singular; 1998: 
36. Goldblum G, Abrahams K, De Waal R, Robertson A. Self Advocacy projects developed by individuals with traumatic brain injury. Poster presentation: The First Regional African AAC Conference, Johannesburg, South Africa; 2004

37. Goldblum, G. Building a house for diversity: Self-advocacy projects developed by individuals with TBI and Aphasia. Presentation at the $2^{\text {nd }}$ Southern African Neurological Rehabilitation Conference (SANRA), Johannesburg, South Africa; February 2005

38. Roosevelt Thomas Jr. R. (with Woodruff M.). The giraffe and the elephant. In: Roosevelt Thomas, Jr. R. (with Woodruff M.). Building a house for diversity. New York: Amacom; 1999: 3-9

39. Bernstein-Ellis E, Elman R. The Book Connection: A life participation book club for individuals with acquired reading impairment, Manual. Oakland, CA: Aphasia Center of California; 2006. Available at www.aphasiacenter.org 40. Albom M. Tuesdays with Morrie. USA: Doubleday; 1997

41. Ross A, Winslow I, Marchant P. Evaluation of communication, life participation and psychological well-being in chronic aphasia: The influence of group intervention. Aphasiology 2006; 20(5): 427-448

42. Avent J, Glista S, Goldblum G. Linking People with Aphasia \& CognitiveCommunication Disabilities using Internet Technologies. Seminar presented at the American Speech-Language-Hearing Association National Annual Convention Chicago; November 2008

43. Goldblum G, Patterson J. Facebook experience: Linking persons with neurogenic communication disorders across continents. Poster presentation, the American Speech-Language-Hearing Association National Annual Convention New 
Orleans; November 2009

44. Sohlberg M. Cognitive Rehabilitation Workshop. Presented at the Centre for Alternative and Augmentative Communication, University of Pretoria; 25-26 February 2003

45. Holland AL. Self-determination and self advocacy: New concepts for aphasic individuals and their partners. In: Conner L, Obler L, eds. Neurobehaviour of language and cognition; 2000

46. Holland AL, Ross R. The power of aphasia groups: Celebrating Roger Ross. In: Elman RJ, ed. Group treatment of neurogenic communication disorders: The expert clinician's approach. $2^{\text {nd }}$ ed.UK: Plural; 2007: 249-256

47 Lightwriter® - Text To Speech Communication Aids: Toby Churchill LTD UK 48. Brown M, Gordon WA, Haddad L. Models for predicting subjective quality of life in individuals with traumatic brain injury. Brain Injury 2000; 14(1): 5-19

49. Whiteneck GG. Measuring what matters: Rehabilitation outcomes. The $44^{\text {th }}$ Annual John Stanley Coulter Lecture. Archives of Physical Medical Rehabilitation 1994; 75: 1073- 1076 